\title{
SOME THEOREMS ON INTEGRAL CURRENTS ${ }^{(1)}$
}

\author{
BY \\ HERBERT FEDERER
}

1. Introduction. A few years ago, in [FF], W. H. Fleming and I began the systematic study of the class of integral currents, which appears to be particularly suitable for the natural formulation and solution of various basic problems in geometric measure theory. The subject of integral currents was developed further in papers by F. J. Almgren, Jr. [A], W. H. Fleming [FL], W. P. Ziemer [Z] and myself [F4], [F5]. Moreover, recent investigations by E. De Giorgi [DG], J. H. Michael [M] and E. R. Reifenberg [R] are closely related to this subject.

The present paper has two aims, to contribute to the basic general study of integral currents, and to establish a connection between this part of analysis and the algebrogeometric theory of complex varieties. The content of $\$ 2$ is a new measure theoretic characterization of integral currents, which makes it easy to recognize complex algebraic varieties as integral cycles. Then $\S 3$ treats the slicing of a normal current $T$ in $X$ by a locally Lipschitzian map $f: X \rightarrow Y$. With almost every point $y$ in $Y$ there is associated, by means of relative differentiation of measures, a current $\langle T, f, y\rangle$ of dimension $\operatorname{dim}(T)-\operatorname{dim}(Y)$, which may be thought of as the slice of $T$ in $f^{-1}\{y\}$. The structure, norm and continuity properties of such slices are examined in detail. This general study seems timely in view of the occurrence of slices in particular cases in [F3], [FF, 3.9], [FL, 1.2], [A], [R] as well as in $\S 4$ of the present paper. That last section was motivated by the classical problem of Plateau, now formulated in terms of minimal currents [FF, §9]. The existence of solutions has been assured, but the determination of the local structure of minimal currents remains a difficult problem. It is now understood that, except in special dimensions, minimal currents will ordinarily have some singularities. One hopes ultimately to prove that almost all the support of a minimal current is a real analytic manifold, and to gain some insight concerning the nature and distribution of the singularities. Considerable progress, though still short of complete success, has been made in [DG], [FL], [R] on the first question, very little on the second. It would certainly be helpful to know many examples of minimal currents with interesting singularities. Examples of this type, and just

Presented to the Society, November 28, 1961, under the title Integral currents in Kähler manifolds and June 1,1962, under the title The behavior of complex algebraic varieties as integral currents; received by the editors June 25, 1963.

(1) This work was supported in part by a research grant from the National Science Foundation. 
about the only ones now known to me, are the complex algebraic varieties. The fact that these varieties are minimal currents is proved in the first part of $\$ 4$, essentially by the classical method of Wirtinger [W], [DR2]; the details are simplified partly by use of the theory of integral currents, but mainly by an extremely short proof of Wirtinger's inequality $\left({ }^{2}\right)$. Then it is shown that the plane sections of a variety depend continuously, as currents, on the cutting planes. The final theorem asserts that one of the algebraic geometer's ways of describing a singular point, by means of a tangent cone whose irreducible components occur with certain intersection multiplicities, is equivalent, for complex varieties, to the purely measure theoretic device used in the theory of minimal currents.

For use this paper we readopt without change the terminology and notation introduced in [FF].

2. A characterization of integral currents. It was proved in [FF, 8.14] that every $k$ dimensional rectifiable current, whose boundary has finite mass, is an integral current. Here it is shown that this condition on the boundary may be lifted on a closed set of $k-1$ dimensional measure 0 .

By $N(f, A, y)$ we mean the number (possibly $\infty$ ) of elements of $A \cap f^{-1}\{y\}$.

2.1 LEMMA. If $T$ is a $k$ dimensional rectifiable current in $R^{n}, B$ is an $H^{k}$ measurable subset of $R^{n}, \lambda \in \Lambda(k, n)$ and

then

$$
\boldsymbol{H}^{k}\left[p^{\lambda}(B)\right]=0
$$

$$
T \wedge p^{\lambda *}\left(\omega_{k}\right) \cap B=0 .
$$

Proof. We know from [FF, 8.16] that $\boldsymbol{H}^{k}$ almost all of the set

$$
A=\left\{z: \Theta^{k}(\|T\|, z)>0\right\}
$$

is contained in the union of a countable family of $k$ dimensional regular submanifolds of class 1 , with tangent spaces spanned by $\vec{T}$. It follows from the classical area formula $[\mathrm{F} 1,5.9]$ that

$$
\int_{A \cap B}\left|p^{\lambda *}\left(\omega_{k}\right)[\vec{T}(z)]\right| d H^{k} z=\int_{R^{k}} N\left(p^{\lambda}, A \cap B, y\right) d H^{k} y .
$$

Since $H^{k}\left[p^{\lambda}(B)\right]=0$, the right integral equals 0 , hence

$$
p^{\lambda *}\left(\omega_{k}\right)[\vec{T}(z)]=0 \text { for } H^{k} \text { almost all } z \text { in } A \cap B .
$$

Therefore, if $\phi \in E^{0}\left(R^{n}\right)$, then

$$
\left[T \wedge p^{\lambda *}\left(\omega_{k}\right) \cap B\right](\phi)=\int_{A \cap B} \phi(z) p^{\lambda *}\left(\omega_{k}\right)[\vec{T}(z)] \Theta^{k}(\|T\|, z) d H^{k} z=0 .
$$

(2) Wirtinger's inequality (Theorem 4.1) involves only linear algebra, and may be read independently from the rest of this paper. 
2.2 TheOREM. Suppose $T \in E_{k}\left(R^{n}\right)$ and $U, V \in E_{k-1}\left(R^{n}\right)$, with

$$
\partial T=U+V \text {. }
$$

If $T$ is rectifiable, $M(U)<\infty$ and $\boldsymbol{H}^{k-1}(\mathrm{spt} V)=0$, then $M(\partial T)<\infty$; hence $T \in I_{k}\left(R^{n}\right)$.

Proof. Assuming that $f \in E^{0}\left(R^{n}\right), \mu \in \Lambda(k-1, n)$ and

$$
\phi=f \wedge p^{\mu *}\left(\omega_{k-1}\right) \in E^{k-1}\left(R^{n}\right),
$$

we shall verify that $|(\partial T)(\phi)| \leqq M(U) \cdot M(\phi)$.

Let $S=p^{\mu}(\operatorname{spt} V)$ and choose $\psi_{1}, \psi_{2}, \psi_{3}, \cdots \in E^{0}\left(R^{k-1}\right)$ such that

$$
\operatorname{spt} \psi_{j} \subset R^{k-1}-S, 0 \leqq \psi_{j}(y) \leqq 1 \text { for } y \in R^{k-1},
$$

for each positive integer $j$, and

$$
\lim _{j \rightarrow \infty} \psi_{j}(y)=1 \text { for } y \in R^{k-1}-S .
$$

Since $d \psi_{j} \wedge \omega_{k-1}=0$, hence $d\left(\psi_{j} \circ p^{\mu}\right) \wedge p^{\mu *}\left(\omega_{k-1}\right)=0$, one obtains

$$
\begin{aligned}
T\left[\left(\psi_{j} \circ p^{\mu}\right) \wedge d \phi\right] & =T\left[d\left[\left(\psi_{j} \circ p^{\mu}\right) \wedge \phi\right]\right]=(\partial T)\left[\left(\psi_{j} \circ p^{\mu}\right) \wedge \phi\right] \\
& =U\left[\left(\psi_{j} \circ p^{\mu}\right) \wedge \phi\right],
\end{aligned}
$$

because $\operatorname{spt}\left(\psi_{j} \circ p^{\mu}\right) \cap \operatorname{spt}(V)$ is empty.

Next observe that $d \phi$ is the sum of $n-k+1$ forms of the type

$$
D_{i} f \wedge d X^{i} \wedge p^{\mu *}\left(\omega_{k-1}\right)= \pm D_{i} f \wedge p^{\lambda *}\left(\omega_{k}\right)
$$

where $i \in\{1, \cdots, n\}-\operatorname{rng} \mu, \lambda \in \Lambda(k, n), \operatorname{rng} \lambda=\{i\} \cup \operatorname{rng} \mu$. For each such $i$ and $\lambda$ we can factor $p^{\mu}=p^{v} \circ p^{\lambda}$, where $v \in \Lambda(k-1, k)$; if $B=\left(p^{\mu}\right)^{-1}(S)$, then

$$
H^{k}\left[p^{\lambda}(B)\right]=H^{k}\left[\left(p^{\eta}\right)^{-1}(S)\right]=0,
$$

and Lemma 2.1 implies that $T \wedge p^{\lambda *}\left(\omega_{k}\right) \cap B=0$; inasmuch as

we find that

$$
\lim _{j \rightarrow \infty}\left(\psi_{j} \circ p^{\mu}\right)(z)=1 \text { for } z \in R^{n}-B,
$$

$$
T\left[D_{i} f \wedge p^{\lambda *}\left(\omega_{k}\right)\right]:=\lim _{j \rightarrow \infty} T\left[\left(\psi_{j} \circ p^{\mu}\right) \wedge D_{i} f \wedge p^{\lambda *}\left(\omega_{k}\right)\right] .
$$

Summation with respect to $i$ yields

$$
\begin{aligned}
T(d \phi) & =\lim _{j \rightarrow \infty} T\left[\left(\psi_{j} \circ p^{\mu}\right) \wedge d \phi\right] \\
& =\lim _{j \rightarrow \infty} U\left[\left(\psi_{j} \circ p^{\mu}\right) \wedge \phi\right],
\end{aligned}
$$

whence $|T(d \phi)| \leqq M(U) \cdot M(\phi)$. 
Thus we have shown that $M(\partial T)<\infty$. Reference to [FF, 8.14] completes the proof.

3. Slices of normal currents. The beginning of this section deals with the problem of extending the definition and elementary properties of $T \wedge f^{*}(\phi)$ from the case where $f$ is of class $\infty$ to the case where $f$ is locally Lipschitzian. Then the slices $\langle T, f, y\rangle$, into which a map $f$ cuts a current $T$ in its domain, are introduced through a procedure motivated by the coarea formula $[F .3,3.1]$ and the proof of [FF, 3.9]. A general treatment of the behavior of these slices, with respect to various norms and transformations, is followed by two theorems which show that under certain special conditions the slices vary continuously.

If $\phi$ is a differential form and $E$ is a set, then $\phi \cap E$ denotes the product of $\phi$ and the characteristic function of $E$.

3.1 Definition. With each locally Lipschitzian map $f: X \rightarrow Y$, of Riemannian manifolds of class $\infty$, we assnciate the commutative diagram

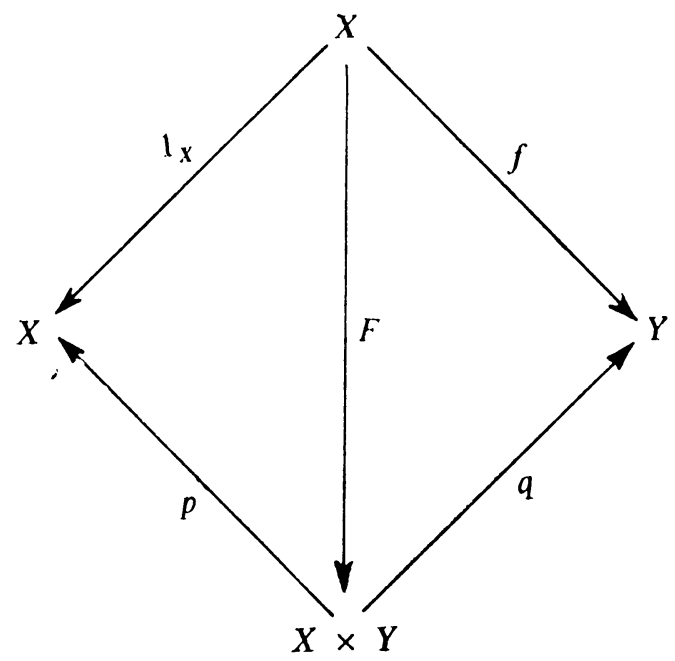

where $p(x, y)=x, q(x, y)=y, F(x)=(x, f(x))$ for $x \in X, y \in Y$. Then we define

$$
\langle T, f, \phi\rangle=p_{*}\left[F_{*}(T) \wedge q^{*}(\phi)\right] \in E_{k-i}(X)
$$

whenever $k \geqq i, T \in N_{k}(X)$ and $\phi$ is a bounded Baireform of degree $i$ on $Y$.

3.2 THEOREM. Under the conditions of 3.1 the following statements hold:

(1) In case $f$ is of class $\infty$, then

$$
\langle T, f, \phi\rangle=T \wedge f^{*}(\phi) .
$$

(2) If $\phi \in E^{i}(Y)$ and $f_{1}, f_{2}, f_{3}, \cdots$ are maps of class $\infty$ which are equi-Lipschitzian and converge uniformly to $f$, on $\operatorname{spt} T$, then 


$$
\lim _{j \rightarrow \infty} F\left[T \wedge f_{j}^{*}(\phi)-\langle T, f, \phi\rangle\right]=0
$$

(3) If $\phi_{1}, \phi_{2}, \phi_{3}, \cdots$ are Baire forms converging boundedly to $\phi$, then

$$
\lim _{j \rightarrow \infty}\left\langle T, f, \phi_{j}\right\rangle=\langle T, f, \phi\rangle \text {. }
$$

(4) For each $\psi \in E^{k-i}(X)$,

$$
\langle T, f, \phi\rangle(\psi)=(-1)^{i(k-i)}\left[f_{*}(T \wedge \psi)\right](\phi) .
$$

(5) In case $k>i$ and $\phi \in E^{i}(Y)$, then

$$
\partial\langle T, f, \phi\rangle=(-1)^{i}\langle\partial T, f, \phi\rangle+(-1)^{i+1}\langle T, f, d \phi\rangle .
$$

(6) In case $k>i=\operatorname{dim} Y$, then $\partial\langle T, f, \phi\rangle=(-1)^{i}\langle\partial T, f, \phi\rangle$.

(7) If either $\phi \in E^{i}(Y)$ or $i=\operatorname{dim} Y$, then $\langle T, f, \phi\rangle \in N_{k-i}(X)$.

(8) If $\lambda$ is a Lipschitz constant for $f$ on spt $T$, then

$$
M(\langle T, f, \phi\rangle) \leqq\left(\begin{array}{c}
k \\
i
\end{array}\right) \lambda^{i}\left[f_{*}(\|T\|)\right](\|\phi\|) .
$$

In case $\phi(y)$ is simple for $y \in Y$, the factor

$$
\left(\begin{array}{c}
k \\
i
\end{array}\right)
$$

may be omitted.

(9) For every bounded Baire function $\psi: Y \rightarrow R$,

$$
\langle T, f, \phi \wedge \psi\rangle=\langle T, f, \phi\rangle \wedge(\psi \circ f) .
$$

(10) In case $i=0$, then $\langle T, f, \phi\rangle=T \wedge(\phi \circ f)$.

(11) If $q \leqq k-i$ and $\psi \in E^{q}(X)$, then

$$
\langle T \wedge \psi, f, \phi\rangle=(-1)^{i q}\langle T, f, \phi\rangle \wedge \psi .
$$

(12) If $Y=R, a<b, E=\{t: a \leqq t \leqq b\}$ and $\phi: R \rightarrow R$,

$$
\phi(y)=[|t-b|-|t-a|+a-b] /[2(a-b)]
$$

whenever $t \in R$, then

$$
(b-a)^{-1}\left\langle T, f, \omega_{1} \cap E\right\rangle=(\partial T) \wedge(\phi \circ f)-\partial[T \wedge(\phi \circ f)] .
$$

Proof. (1) holds because, for each $\psi \in E^{k-i}(X)$,

$$
\begin{aligned}
\langle T, f, \phi\rangle(\psi) & =\left[F_{*}(T) \wedge q^{*}(\phi)\right]\left[p^{*}(\psi)\right] \\
& =F_{*}(T)\left[q^{*}(\phi) \wedge p^{*}(\psi)\right]=T\left[\left(F^{*} \circ q^{*}\right)(\phi) \wedge\left(F^{*} \circ p^{*}\right)(\psi)\right] \\
& =T\left[f^{*}(\phi) \wedge \psi\right]=\left[T \wedge f^{*}(\phi)\right](\psi) .
\end{aligned}
$$


To verify (2) we note that the maps

$$
F_{j}: X \rightarrow X \times Y, F_{j}(x)=\left(x, f_{j}(x)\right) \text { for } x \in X,
$$

are equi-Lipschitzian and converge uniformly to $F$, on spt $T$, and apply [FF, 3.4].

(3) holds because $M\left[F_{*}(T)\right]<\infty$.

The statements (4)-(11) are readily verified by reference to $[\mathrm{FF}, 2.3,2.4]$ in case $f$ is of class $\infty$ and $\phi \in E^{i}(Y)$, and then extended by approximation. Regarding (8) we observe that, in the smooth case,

$$
M\left[T \wedge f^{*}(\phi)\right] \leqq\left(\begin{array}{c}
k \\
i
\end{array}\right)\|T\|\left(\left\|f^{*}(\phi)\right\|\right) \leqq\left(\begin{array}{c}
k \\
i
\end{array}\right)\|T\|\left[\lambda^{i}(\|\phi\| \circ f)\right] .
$$

To prove (12) we approximate $\phi$ uniformly by a sequence of equi-Lipschitzian functions $\phi_{j} \in E^{0}(R)$ such that

$$
\lim _{j \rightarrow \infty} \phi_{j}^{\prime}(y)=\phi^{\prime}(y) \text { whenever } y \in R-\{a, b\} .
$$

From (5) and (10) we obtain the equations

$$
\left\langle T, f, d \phi_{j}\right\rangle=(\partial T) \wedge\left(\phi_{j} \circ f\right)-\partial\left[T \wedge\left(\phi_{j} \circ f\right)\right]
$$

whose right members clearly converge to the right member of (12). Moreover, if $\psi \in E^{k-1}(U)$, then $f_{*}(T \wedge \psi) \in N_{1}\left(R^{1}\right)$ and [FF, 6.4] implies that the measure $f_{*}(T \wedge \psi) \wedge \omega_{1}$ is absolutely continuous with respect to $L_{1}$; applying (4) we find that

$$
\left\langle T, f, d \phi_{j}\right\rangle(\psi)=(-1)^{k-1} f_{*}(T \wedge \psi)\left[\phi_{j}^{\prime} \omega_{1}\right]
$$

converges to

$$
(-1)^{k-1} f_{*}(T \wedge \psi)\left[(b-a)^{-1} \omega_{1} \cap E\right],
$$

which equals the left member of (12).

3.3 THEOREM. If

$$
X \stackrel{f}{\longrightarrow} Y \stackrel{g}{\longrightarrow} Z
$$

are locally Lipschitzian maps of Riemannian manifolds of class $\infty, k \geqq i$, $T \in N_{k}(X)$ and $\phi$ is a bounded Baire form of degree $i$ on $Z$, then

$$
f_{*}(\langle T, g \circ f, \phi\rangle)=\left\langle f_{*}(T), g, \phi\right\rangle .
$$

In case $g$ is of class $\infty$, then

$$
\langle T, g \circ f, \phi\rangle=\left\langle T, f, g^{*}(\phi)\right\rangle .
$$

3.4 REMARK. Whenever $y \in R^{n}$ and $E_{1}, E_{2}, E_{3}, \cdots$ are subsets of $R^{n}$ such that 


$$
\begin{gathered}
\inf \left\{\operatorname{diam}\left(E_{j}\right)^{-n} L_{n}\left(E_{j}\right): j=1,2,3, \cdots\right\}>0, \\
\lim _{j \rightarrow \infty} \operatorname{diam}\left(E_{j} \cup\{y\}\right)=0,
\end{gathered}
$$

one refers to $E_{1}, E_{2}, E_{3}, \cdots$ as a regular sequence of sets tending to $y$. We shall compute the general derivates of certain measures with respect to such regular sequences [S, Chapter IV].

3.5 Theorem. Suppose $X$ is a Riemannian manifold of class $\infty, f: X \rightarrow R^{n}$ is locally Lipschitzian, $T \in N_{k}(X)$ and $k \geqq n$. For $L_{n}$ almost all $y$ in $R^{n}$ there exists a unique current

$$
\langle T, f, y\rangle \in N_{k-n}(X)
$$

such that, if $E_{1}, E_{2}, E_{3}, \cdots$ is any regular sequence of closed sets tending to $y$, and if

$$
\lambda_{j}=L_{n}\left(E_{j}\right)^{-1}, \quad S_{j}=\lambda_{j}\left\langle T, f, \omega_{n} \cap E_{j}\right\rangle
$$

for $j=1,2,3, \cdots$, then

$$
\langle T, f, y\rangle(\psi)=(-1)^{n(k-n)} \lim _{j \rightarrow \infty} \lambda_{j}\left[f_{*}(T \wedge \psi) \wedge \omega_{n}\right]\left(E_{j}\right)
$$

whenever $\psi \in E^{k-n}(X)$, and

$$
\begin{gathered}
\underset{j \rightarrow \infty}{\limsup \sup _{j} M\left(S_{j}\right)}=\limsup _{j \rightarrow \infty} \lambda_{j}\left[f_{*}\left(\left\|\left\langle T, f, \omega_{n}\right\rangle\right\|\right)\right]\left(E_{j}\right)<\infty, \\
\underset{j \rightarrow \infty}{\lim \sup } M\left(\partial S_{j}\right)= \\
\lim _{j \rightarrow \infty} F\left(S_{j}-\langle T, f, y\rangle\right)=0, \\
\operatorname{spt}\langle T, f, y\rangle \subset(\operatorname{spt} T) \cap f^{-1}\{y\} .
\end{gathered}
$$

Proof. Assuming $X$ separable, let $D$ be a countable $M$ dense subset of $E^{k-n}(X)$. For $L_{n}$ almost all $y$ in $R^{n}$, the measures

$$
f_{*}\left(\left\|\left\langle T, f, \omega_{n}\right\rangle\right\|\right), f_{*}\left(\left\|\left\langle\partial T, f, \omega_{n}\right\rangle\right\|\right),
$$

and all the measures

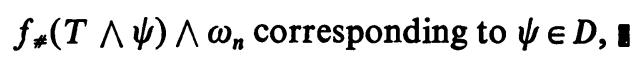

are derivable in the general sense with respect to $L_{n}$.

We now fix such a point $y$ and let $a, b, c(\psi)$ be the values, at $y$, of the general derivates of the above measures.

Considering any regular sequence of closed sets $E_{j}$ tending to $y$, we see from 3.2 (7), (5) that 


$$
\begin{gathered}
S_{j}=\lambda_{j}\left\langle T, f, \omega_{n}\right\rangle \cap f^{-1}\left(E_{j}\right), \\
M\left(S_{j}\right)=\lambda_{j}\left\|\left\langle T, f, \omega_{n}\right\rangle\right\|\left[f^{-1}\left(E_{j}\right)\right] \\
=\lambda_{j} f_{*}\left(\left\|\left\langle T, f, \omega_{n}\right\rangle\right\|\right)\left(E_{j}\right), \\
\partial S_{j}=\lambda_{j}(-1)^{n}\left\langle\partial T, f, \omega_{n}\right\rangle \cap f^{-1}\left(E_{j}\right), \\
M\left(\partial S_{j}\right)=\lambda_{j} f_{*}\left(\left\|\left\langle\partial T, f, \omega_{n}\right\rangle\right\|\right)\left(E_{j}\right), \\
\lim _{j \rightarrow \infty} M\left(S_{j}\right)=a, \lim _{j \rightarrow \infty} M\left(\partial S_{j}\right)=b,
\end{gathered}
$$

and from 3.2 (3) that, for every $\psi \in D$,

$$
\begin{aligned}
& S_{j}(\psi)=(-1)^{n(k-n)} \lambda_{j}\left[f_{*}(T \wedge \psi)\right]\left(\omega_{n} \cap E_{j}\right) \\
&=(-1)^{n(k-n)} \lambda_{j}\left[f_{*}(T \wedge \psi) \wedge \omega_{n}\right]\left(E_{j}\right), \\
& \lim _{j \rightarrow \infty} S_{j}(\psi)=(-1)^{n(k-n)} c(\psi) .
\end{aligned}
$$

It follows that the currents $S_{j}$ converge, with respect to the norm $F$ according to $[\mathrm{FF}, 7.3]$, to a current $S \in N_{k-n}(X)$ for which

$$
S(\psi)=(-1)^{n(k-n)} c(\psi) \text { whenever } \psi \in D .
$$

From this formula we also see that $S$ is independent of the choice of regular sequence, and may be denoted $\langle T, f, y\rangle$.

The last conclusion of the theorem holds because

3.6 Corollary.

$$
\operatorname{spt}\left(S_{j}\right) \subset(\operatorname{spt} T) \cap f^{-1}\left(E_{j}\right)
$$

(1) Whenever $k>n$ and $\langle T, f, y\rangle$ exists, so does

$$
\langle\partial T, f, y\rangle=(-1)^{n} \partial\langle T, f, y\rangle .
$$

(2) Whenever $\psi \in E^{q}(X), q \leqq k-n$ and $\langle T, f, y\rangle$ exists, so does

$$
\langle T \wedge \psi, f, y\rangle=(-1)^{n q}\langle T, f, y\rangle \wedge \psi \text {. }
$$

(3) For every bounded Baire form $\psi$ of degree $k-n$ on $X$,

$$
\left\langle T, f, \omega_{n}\right\rangle(\psi)=\int_{R^{n}}\langle T, f, y\rangle(\psi) d L_{n} y .
$$

(4) $M\left(\left\langle T, f, \omega_{n}\right\rangle\right)=\int_{R^{n}} M(\langle T, f, y\rangle) d L_{n} y$.

(5) For every bounded Baire function $\psi: X \rightarrow R$,

$$
\left\|\left\langle T, f, \omega_{n}\right\rangle\right\|(\psi)=\int_{R^{n}}\|\langle T, f, y\rangle\|(\psi) d L_{n} y .
$$

(6) If $u: R^{n} \rightarrow R$ is a bounded Baire function, then 


$$
\left\langle T, f, u \omega_{n}\right\rangle(\gamma)=\int_{R^{n}}\langle T, f, y\rangle(\gamma) \cdot u(y) d L_{n} y
$$

whenever $\gamma \in E^{k-n}(X)$, and

$$
M\left(\left\langle T, f, u \omega_{n}\right\rangle\right)=\int_{R^{n}} M(\langle T, f, y\rangle) \cdot|u(y)| d L_{n} y .
$$

(7) In case $n=1$ and $\langle T, f, y\rangle$ exists, then

$$
\langle T, f, y\rangle=(\partial T) \cap\{x: f(x)>y\}-\partial(T \cap\{x: f(x)>y\}) .
$$

(8) Suppose $V$ and $W$ are open subsets of $R^{n}, g$ is a diffeomorphism of class $\infty$ mapping $V$ onto $W, f(\operatorname{spt} T) \subset V$, and $y \in V$. If $\langle T, f, y\rangle$ exists, so does

$$
\langle T, g \circ f, g(y)\rangle=\varepsilon\langle T, f, y\rangle,
$$

where $\varepsilon=\operatorname{det} D g(y) /|\operatorname{det} D g(y)|$.

(9) In case $X$ is an open subset of $R^{n}$, then

$$
\int_{R^{n}} F(\langle T, f, y\rangle) d L_{n} y \leqq F(T) .
$$

Proof. (1) and (2) are obvious. To obtain (3) in case $\psi \in E^{k-n}(X)$, we note that $f_{*}(T \wedge \psi) \in N_{n}\left(R^{n}\right)$ and infer from $[\mathrm{FF}, 6.4]$ that the measure $f_{*}(T \wedge \psi) \wedge \omega_{n}$ is absolutely continuous with respect to $L_{n}$; looking up its derivate in the statement of our theorem, we find that

$$
\begin{aligned}
\int_{R^{n}}\langle T, f, y\rangle(\psi) d L_{n} y & =(-1)^{n(k-n)}\left[f_{*}(T \wedge \psi) \wedge \omega_{n}\right]\left(R^{n}\right) \\
& =(-1)^{n(k-n)}\left[f_{*}(T \wedge \psi)\right]\left(\omega_{n}\right)=\left\langle T, f, \omega_{n}\right\rangle(\psi)
\end{aligned}
$$

by $3.2(3)$. Next we infer from (3) that

$$
M\left(\left\langle T, f, \omega_{n}\right\rangle\right) \leqq \int_{R^{n}} M(\langle T, f, y\rangle) d L_{n} y ;
$$

moreover our theorem shows that the above integrand is dominated, $L_{n}$ almost everywhere, by the derivate of $f_{*}\left(\left\|\left\langle T, f, \omega_{n}\right\rangle\right\|\right)$; hence the integral does not exceed

$$
\begin{aligned}
f_{*}\left(\left\|\left\langle T, f, \omega_{n}\right\rangle\right\|\right)\left(R^{n}\right) & =\left\|\left\langle T, f, \omega_{n}\right\rangle\right\|(X) \\
& =M\left(\left\langle T, f, \omega_{n}\right\rangle\right) .
\end{aligned}
$$

Thus we have verified (4), from which (5) results for $\psi \in E^{0}(X)$ through replacement of $T$ by $T \wedge \psi$, and then for general $\psi$ through approximation.

The first part of (6) is obtained from (3) with $\psi=\gamma \cdot(u \circ f)$, the second from (5) with $\psi=u \circ f$.

To prove (7) we consider the sequence of intervals 


$$
E_{j}=\left\{t: y \leqq t \leqq y+j^{-1}\right\}
$$

defining $\phi_{j}$ as in 3.2 (11) we find that

$$
S_{j}=j\left\langle T, f, \omega_{1} \cap E_{j}\right\rangle=(\partial T) \wedge\left(\phi_{j} \circ f\right)-\partial\left[T \wedge\left(\phi_{j} \circ f\right)\right],
$$

and observe that the functions $\phi_{j} \circ f$ converge boundedly to the characteristic function of $\{x: f(x)>y\}$.

As for (8), we note that the regular sequences tending to $y$ correspond under $g$ to the regular sequences tending to $g(y)$. Letting

$$
\mu_{j}=L_{n}\left[g\left(E_{j}\right)\right]^{-1}, \quad \sigma_{j}=\mu_{j}\left\langle T, g \circ f, \omega_{n} \cap g\left(E_{j}\right)\right\rangle,
$$

we see from 3.3 and 3.2 (9) that

hence

$$
\begin{aligned}
\sigma_{j} & =\mu_{j}\left\langle T, f, g^{*}\left[\omega_{n} \cap g\left(E_{j}\right)\right]\right\rangle \\
& =\mu_{j}\left\langle T, f,(\operatorname{det} D g) \omega_{n} \cap E_{j}\right\rangle, \\
\sigma_{j}-\varepsilon S_{j} & =\lambda_{j}\left\langle T, f,\left(\mu_{j} \lambda_{j}^{-1} \operatorname{det} D g-\varepsilon\right) \omega_{n} \cap E_{j}\right\rangle \\
& =S_{j} \wedge\left[\left(\mu_{j} \lambda_{j}^{-1} \operatorname{det} D g-\varepsilon\right) \cap E_{j}\right] \circ f,
\end{aligned}
$$

$$
\boldsymbol{M}\left(\sigma_{j}-\varepsilon S_{j}\right) \leqq M\left(S_{j}\right) \cdot \boldsymbol{M}\left[\left(\mu_{j} \lambda_{j}^{-1} \operatorname{det} D g-\varepsilon\right) \cap E_{j}\right]
$$

approaches 0 as $j$ approaches $\infty$.

In verifying (9) we may assume, possibly modifying $f$ on $X-\operatorname{spt} T$ and performing a Lipschitzian extension, that $X$ is convex. Then we use [FF, 7.2] to choose $K \in N_{k+1}(X)$ so that

infer from (1) that

$$
\boldsymbol{F}(T)=\boldsymbol{M}(T-\partial K)+\boldsymbol{M}(K),
$$

$$
\langle T, f, y\rangle=\langle T-\partial K, f, y\rangle+(-1)^{n} \partial\langle K, f, y\rangle
$$

for $L_{n}$ almost all $y$, and apply (4) to obtain

$$
\begin{aligned}
\int_{R^{n}} \boldsymbol{F}(\langle T, f, y\rangle) d L_{n} y & \leqq \int_{R^{n}}[M(\langle T-\partial K, f, y\rangle)+\boldsymbol{M}(\langle K, f, y\rangle)] d L_{n} y \\
& \leqq M(T-\partial K)+\boldsymbol{M}(K)=\boldsymbol{F}(T) .
\end{aligned}
$$

3.7 REMARK. In view of $3.6(8)$ one may define $\langle T, f, y\rangle$ not only for maps $f$ into $R^{n}$, but for maps into any oriented $n$ dimensional manifold $Y$ of class $\infty$. When $Y$ is given a Riemannian metric, inducing the Hausdorff measure $\boldsymbol{H}^{n}$ over $Y$, then $\langle T, f, y\rangle$ exists for $H^{n}$ almost all $y$. All statements of 3.6 remain true when $L_{n}$ and $\omega_{n}$ are replaced by $\boldsymbol{H}^{n}$ and the orienting unit $n$ form of $Y$.

3.8 REMARK. It follows from 3.6 (9) and Fatou's lemma that, if $T_{1}, T_{2}, T_{3}, \cdots$ are normal currents which converge to $T$ with respect to the norm $F$, then 


$$
\underset{j \rightarrow \infty}{\lim \inf } F\left[\left\langle T_{j}, f, y\right\rangle-\langle T, f, y\rangle\right]=0
$$

for $L_{n}$ almost all $y$ in $R^{n}$.

3.9 THEOREM. Under the conditions of 3.3, if $Z$ is oriented, $z \in Z$, and $\langle T, g \circ f, z\rangle$ exists, so does $\left\langle f_{*}(T), g, z\right\rangle=f_{\#}(\langle T, g \circ f, z\rangle)$.

Proof. If $Z=R^{n}$ and $E_{1}, E_{2}, E_{3}, \cdots$ is a regular sequence of closed sets tending to $z$, with $\lambda_{j}=L_{n}\left(E_{j}\right)^{-1}$, then $\lambda_{j}\left\langle f_{*}(T), g, \omega_{n} \cap E_{j}\right\rangle=f_{*}\left(\lambda_{j}\left\langle T, g \circ f, \omega_{n} \cap E_{j}\right\rangle\right)$.

3.10 THEOREM. If the conditions of 3.1 hold and $y \in Y=R^{n}$, then

$$
\begin{aligned}
\left\langle F_{\#}(T), q, y\right\rangle & =F_{*}(\langle T, f, y\rangle), \\
\langle T, f, y\rangle & =p_{\#}\left(\left\langle F_{*}(T), q, y\right\rangle\right) .
\end{aligned}
$$

Proof. Since $f=q \circ F$, (1) holds whenever $\langle T, f, y\rangle$ exists. On the other hand 3.1 and 3.2 (1) yield the equations $\lambda_{j}\left\langle T, f, \omega_{n} \cap E_{j}\right\rangle=p_{*}\left(\lambda_{j}\left\langle F_{*}(T), q, \omega_{n} \cap E_{j}\right\rangle\right)$, which show that (2) holds whenever $\left\langle F_{\#}(T), q, y\right\rangle$ exists.

3.11 THEOREM. Consider a commutative diagram

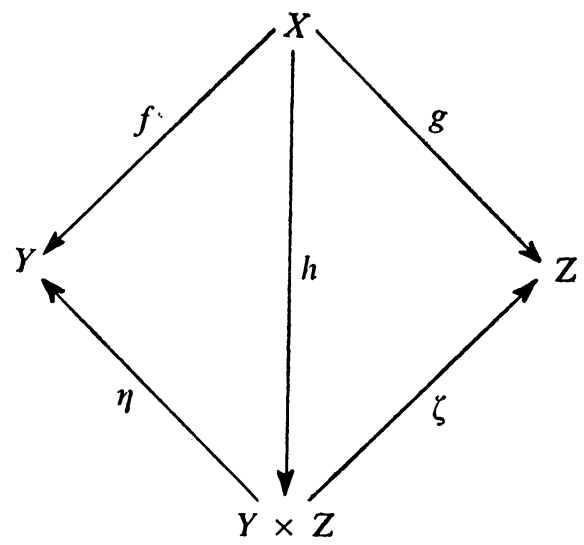

of locally Lipschitzian maps of Riemannian manifolds, where $\eta$ and $\zeta$ are the projections, and suppose $T \in N_{k}(X), k \geqq m+n$.

(1) If $\phi \in E^{m}(Y), \psi \in E^{n}(Z)$, then

$$
\begin{aligned}
\left\langle T, h, \eta^{*}(\phi) \wedge \zeta^{*}(\psi)\right\rangle & =\langle\langle T, f, \phi\rangle, g, \psi\rangle \\
& =(-1)^{m n}\langle\langle T, g, \psi\rangle, f, \phi\rangle .
\end{aligned}
$$

(2) In case $Z=R^{n}$ and $\phi \in E^{m}(Y)$, then

$$
\langle\langle T, f, \phi\rangle, g, z\rangle=(-1)^{m n}\langle\langle T, g, z\rangle, f, \phi\rangle
$$

for $L_{n}$ almost all $z$. 
(3) In case $Y=R^{m}$ and $Z=R^{n}$, then

$$
\begin{aligned}
\langle T, h,(y, z)\rangle & =\langle\langle T, f, y\rangle, g, z\rangle \\
& =(-1)^{m n}\langle\langle T, g, z\rangle, f, y\rangle
\end{aligned}
$$

for $L_{m+n}$ almost all $(y, z)$ in $R^{m} \times R^{n}$.

Proof. One readily verifies (1), first in case $h$ is of class $\infty$, then in general using 3.2 (2). Similarly (2) follows from 3.6 (2) in case $f$ is of class $\infty$, and is extended with the help of 3.6 (9).

In proving (3) we apply (1) and (2) with $\phi=u \omega_{m}$ and $\psi=v \omega_{n}$, where $u \in E^{0}\left(R^{m}\right)$ and $v \in E^{0}\left(R^{n}\right)$, together with $3.6(6)$ to obtain, for each $\gamma \in E^{k-m-n}(X)$, the equations

$$
\begin{aligned}
\int_{R^{m+n}} & \langle T, h,(y, z)\rangle(\gamma) u(y) v(z) d L_{m+n}(y, z) \\
& =\int_{R^{n}}\left\langle\left\langle T, f, u \omega_{m}\right\rangle, g, z\right\rangle(\gamma) v(z) d L_{n} z \\
& =\int_{R^{n}} \int_{R^{m}}(-1)^{m n}\langle\langle T, g, z\rangle, f, y\rangle(\gamma) u(y) d L_{m} y v(z) d L_{n} z .
\end{aligned}
$$

The equality of the first and third members of (3) now results from Fubini's theorem and the arbitrary nature of $u$ and $v$.

3.12 THEOREM. If $f: X \rightarrow R^{n}$ is locally Lipschitzian, $k \geqq n$ and $T \in I_{k}(X)$, then

$$
\langle T, f, y\rangle \in I_{k-n}(X) \text { for } L_{n} \text { almost all } y .
$$

Proof. In case $n=1$ the conclusion follows from 3.6 (7) and [FF, 8.14 and 3.9(3)]. The general case may be obtained by induction using $3.11(3)$.

3.13 RemarK. Assuming that $T \in I_{k}(X), k \geqq n$ and $f: X \rightarrow R^{n}$ is of class $\infty$, one may compute the currents $T \wedge f^{*}\left(\omega_{n}\right)$ and $S_{y}=\langle T, f, y\rangle$ as follows:

For $\|T\|$ almost all $x$ in $X, \vec{T}(x)$ exists and is a simple unit $k$ vector. Let $J(x)$ be the norm of the restriction of $f^{*}\left(\omega_{n}\right)$ to the $k$ space of $\vec{T}(x)$. In case $J(x) \neq 0$, there exist a unique simple unit $k-n$ vector $w(x)$ and a unique simple unit $n$ vector $v(x)$ such that the $k-n$ space of $w(x)$ is the intersection of the kernel of $D f(x)$ with the $k$ space of $\vec{T}(x)$, and

$$
\vec{T}(x)=v(x) \wedge w(x), \quad J(x)=f^{*}\left(\omega_{n}\right)[v(x)] .
$$

These definitions immediately imply the first two of the three statements:

(1) If $\phi \in E^{k-n}(X)$, then

$$
\left[T \wedge f^{*}\left(\omega_{n}\right)\right](\phi)=\int J(x) \cdot \phi(x)[w(x)] d\|T\| x .
$$


(2) If $\psi: X \rightarrow R$ is a bounded Baire function, then

$$
\left\|T \wedge f^{*}\left(\omega_{n}\right)\right\|(\psi)=\int \psi(x) J(x) d\|T\| x .
$$

(3) For $L_{n}$ almost all $y$ in $R^{n}$ it is true that

$$
\begin{aligned}
\vec{S}_{y}(x) & =w(x) \text { for }\left\|S_{y}\right\| \text { almost all } x \text { in } X, \\
\Theta^{k-n}\left(\left\|S_{y}\right\|, x\right) & =\Theta^{k}(\|T\|, x) \text { for } H^{k-n} \text { almost all } x \text { in } f^{-1}\{y\} .
\end{aligned}
$$

To verify (3), we take any $g \in E^{0}\left(R^{n}\right)$ and $\phi \in E^{k-n}(X)$, let

$$
h(x)=g[f(x)] \cdot \phi(x)[w(x)] \text { for } x \in X,
$$

and use 3.6 (3), the above (1) and (2), and 3.6(5) to obtain

$$
\begin{aligned}
\int g(y) S_{y}(\phi) d L_{n} y & =\left\langle T, f, \omega_{n}\right\rangle[(g \circ f) \cdot \phi] \\
& =\|T\|(J \cdot h)=\left\|\left\langle T, f, \omega_{n}\right\rangle\right\|(h) \\
& =\int\left\|S_{y}\right\|(h) d L_{n} y \\
& =\int g(y) \int \phi(x)[w(x)] d\left\|S_{y}\right\| x d L_{n} y .
\end{aligned}
$$

Consequently, for $L_{n}$ almost all $y$,

$$
S_{y}(\phi)=\int \phi(x)[w(x)] d\left\|S_{y}\right\| x \quad \text { whenever } \phi \in E^{k-n}(X),
$$

whence the first conclusion follows. To obtain the second, we take any $\gamma \in E^{0}(X)$ and infer from 3.13, [FF, 8.16], 3.6 (5), the above (2), the coarea formula [F3, 3.1] and the generalized Eilenberg inequality $[F 2,3.2]$ that

$$
\begin{aligned}
\int g(y) & \int \gamma(x) \Theta^{k-n}\left(\left\|S_{y}\right\|, x\right) d H^{k-n} x d L_{n} y \\
& =\int\left\|S_{y}\right\|[(g \circ f) \cdot \gamma] d L_{n} y=\left\|T \wedge f^{*}\left(\omega_{n}\right)\right\|[(g \circ f) \cdot \gamma] \\
& =\int g[f(x)] \gamma(x) J(x) d\|T\| x \\
& =\int g[f(x)] \gamma(x) J(x) \Theta^{k}(\|T\|, x) d H^{k} x \\
& =\int g(y) \int_{f^{-1}\{y\}} \gamma(x) \Theta^{k}(\|T\|, x) d H^{k-n} x d L_{n} y,
\end{aligned}
$$

and use the arbitrary choice of $g$ and $\gamma$ to complete the proof. 
3.14 THEOREM. If $h: R \times X \rightarrow R^{n}$ is locally Lipschitzian, $k \geqq n, T \in N_{k}(X)$, $a<b, J=\{t: a<t<b\}$ and

$$
f(x)=h(a, x), \quad g(x)=h(b, x), \quad q(t, x)=x
$$

whenever $x \in X$ and $t \in R$, then:

(1) For every bounded Baire form $\phi: R^{n} \rightarrow \bigwedge^{n}\left(R^{n}\right)$,

$$
\langle T, g, \phi\rangle-\langle T, f, \phi\rangle=q_{*}\left[\langle J \times \partial T, h, \phi\rangle+(-1)^{n} \partial\langle J \times T, h, \phi\rangle\right] .
$$

(2) For $L_{n}$ almost all $y$ in $R^{n}$,

$$
\langle T, g, y\rangle-\langle T, f, y\rangle=q_{*}\left[\langle J \times \partial T, h, y\rangle+(-1)^{n} \partial\langle J \times T, h, y\rangle\right] .
$$

Proof. To prove (1), we apply 3.2 (6) and 3.3, with $A(x)=(a, x)$ and $B(x)=(b, x)$ for $x \in X$, to obtain

$$
\begin{aligned}
q_{*}\left[(-1)^{n} \partial\langle J \times T, h, \phi\rangle\right]= & q_{*}[\langle\partial(J \times T), h, \phi\rangle] \\
= & q_{*}\left[\left\langle B_{*}(T), h, \phi\right\rangle-\left\langle A_{*}(T), h, \phi\right\rangle\right. \\
& -\langle J \times \partial T, h, \phi\rangle] \\
= & \langle T, g, \phi\rangle-\langle T, f, \phi\rangle-q_{*}[\langle J \times \partial T, h, \phi\rangle] .
\end{aligned}
$$

Then we deduce (2) from (1), with $\phi=\omega_{n} \cap E_{j}$.

3.15 ThEOREM('). If $k \geqq n, T \in N_{k}(X), f: X \rightarrow R^{n}$ has the Lipschitz constant $\lambda$ on spt $T$, and there exists a finite positive number $\mu$ such that

$$
(\|\partial T\|+\|T\|)\left[f^{-1}(E)\right] \leqq \mu L_{n}(E) \text { whenever } E \subset R^{n},
$$

then the following two statements hold for all $y$ and $z$ in $R^{n}$ :

(1) $\langle T, f, y\rangle \in N_{k-n}(X), N(\langle T, f, y\rangle) \leqq \lambda^{n} \mu$,

(2) $F(\langle T, f, y+z\rangle-\langle T, f, y\rangle) \leqq|z| \lambda^{n-1} \mu$.

Proof. Using $3.2(8)$ we see that

$$
M\left(\left\langle T, f, \omega_{n} \cap E\right\rangle\right)+M\left(\left\langle\partial T, f, \omega_{n} \cap E\right\rangle\right) \leqq \lambda^{n} \mu L_{n}(E)
$$

for every Borel set $E \subset R^{n}$, hence (1) holds whenever $\langle T, f, y\rangle$ exists.

Next suppose $0 \neq z \in R^{n}$ and let $J=\left\{t: 0<t<|z| \lambda^{-1}\right\}$,

$$
g(x)=f(x)-z, \quad h(t, x)=f(x)-t \lambda|z|^{-1} z
$$

whenever $t \in R, x \in X$. For every Borel set $E \subset R^{n}$ we know from 3.3 that

$$
\left\langle T, g, \omega_{n} \cap E\right\rangle=\left\langle T, f, \omega_{n} \cap(E+z)\right\rangle,
$$

and use $3.14(1), 3.2(8)$ to infer that

(3) This theorem may be used to verify the continuity of the map foccurring in [A, 3.3]. 


$$
\begin{aligned}
\boldsymbol{F}[\langle & \left.\left\langle, f, \omega_{n} \cap(E+z)\right\rangle-\left\langle T, f, \omega_{n} \cap E\right\rangle\right] \\
& \leqq M\left(\left\langle J \times \partial T, h, \omega_{n} \cap E\right\rangle\right)+M\left(\left\langle J \times T, h, \omega_{n} \cap E\right\rangle\right) \\
& \leqq \lambda^{n}(\|J \times \partial T\|+\|J \times T\|)\left[h^{-1}(E)\right] \\
& \leqq \lambda^{n}(\|J\| \times\|\partial T\|+\|J\| \times\|T\|)\left[h^{-1}(E)\right] \\
& =\lambda^{n} \int_{J}(\|\partial T\|+\|T\|)\left[\left\{x:(t, x) \in h^{-1}(E)\right\}\right] d L_{1} t \\
& =\lambda^{n} \int_{J}(\|\partial T\|+\|T\|)\left[f^{-1}\left(E+t \lambda|z|^{-1} z\right)\right] d L_{1} t \\
& \leqq|z| \lambda^{n-1} \mu L_{n}(E) .
\end{aligned}
$$

From the preceding inequality we see that (2) holds whenever $\langle T, f, y+z\rangle$ and $\langle T, f, y\rangle$ both exist.

Now it follows immediately that $\langle T, f, y\rangle$ exists for every $y$ in $R^{n}$.

3.16 REMARK. If $k \geqq n, T \in N_{k}(X)$ and the maps $f: X \rightarrow R^{n}, g: X \rightarrow R^{n}$ have the common Lipschitz constant $\lambda$ on $\operatorname{spt} T$, then

$$
\int_{R^{n}} F(\langle T, g, y\rangle-\langle T, f, y\rangle) d L_{n} y \leqq \lambda^{n-1}(\|\partial T\|+\|T\|)[|f-g|] .
$$

This may be verified by applying 3.14 (2) to the linear homotopy $h$ from $f$ to $g$, with $J=\{t: 0 \leqq t \leqq 1\}$. In case $f$ and $g$ are of class $\infty$ one readily computes

$$
h^{\#}\left(\omega_{n}\right)=d p \wedge q^{*}(\sigma)+\psi \wedge q^{*}(\tau),
$$

where $p: R \times X \rightarrow R, \quad p(t, x)=t$, and

$$
\begin{gathered}
\sigma \in E^{n-1}(X), \tau \in E^{n}(X), \psi \in E^{0}(R \times X), \\
\|\sigma(x)\| \leqq \lambda^{n-1}|f(x)-g(x)| \text { whenever } x \in \operatorname{spt} T ;
\end{gathered}
$$

therefore, if $S$ is either $T$ or $\partial T$, then

$$
M\left(\left\langle J \times S, h, \omega_{n}\right\rangle\right) \leqq \lambda^{n-1}\|S\|(|f-g|) .
$$

This inequality clearly extends to the general Lipschitzian case, and then 3.6 (5) completes the proof.

3.17 TheOREM. Suppose $f: X \rightarrow R^{n}$ is of class $\infty, T \in I_{n}(X)$, spt $T-\operatorname{spt} \partial T$ is locally connected, $\mu$ and $v$ are positive integers,

$$
\Theta^{k}(\|T\|, x) \leqq \mu \text { whenever } x \in X,
$$

$(\operatorname{spt} T) \cap f^{-1}\{y\}$ has at most $v$ elements whenever $y \in R^{n}-f(\operatorname{spt} \partial T)$.

Let $G$ be the class of all nonempty connected open subsets of $R^{n}-f(\operatorname{spt} \partial T)$; 
for each $W \in G$ let $\Gamma(W)$ be the set of all components of (spt $T) \cap f^{-1}(W)$; also let

$$
H=\bigcup\{\Gamma(W): W \in G\}
$$

Then:

(1) For each $V \in \Gamma(W)$ there exists an integer $\Delta(V)$ such that

$$
f_{\#}(T \cap V)=\Delta(V) \cdot W .
$$

(2) If $V \in \Gamma(W)$ and $\Delta(V) \neq 0$, then $f(V)=W$.

(3) $\Gamma(W) \cap\{V: \Delta(V) \neq 0\}$ has at most $v$ elements.

(4) If $W \subset W^{\prime}$ belong to $G$ and $V^{\prime} \in \Gamma\left(W^{\prime}\right)$, then

$$
\Delta\left(V^{\prime}\right)=\sum_{V^{\prime}, V \in \Gamma(W)} \Delta(V) .
$$

(5) $H$ is a base for the relative topology of $\operatorname{spt} T-\operatorname{spt} \partial T$.

(6) If $x \in \operatorname{spt} T-\operatorname{spt} \partial T$, then $\Delta(V)$ has the same value, hereafter denoted $\Delta(x)$,for all sufficiently small neighborhoods $V$ of $x$, belonging to $H$.

(7) For every Borel set $E \subset R^{n}-f(\operatorname{spt} \partial T)$,

$$
M\left(\left\langle T, f, \omega_{n} \cap E\right\rangle\right) \leqq \mu \nu L_{n}(E) .
$$

(8) For $y \in R^{n}-f(\operatorname{spt} \partial T)$ and $\psi \in E^{0}(X)$,

$$
\langle T, f, y\rangle(\psi)=\sum_{x \in f^{-1}(y)} \Delta(x) \cdot \psi(x) .
$$

(9) $\langle T, f, \cdot\rangle$ is $M$ bounded and $\boldsymbol{F}$ continuous on $R^{n}-f(\operatorname{spt} \partial T)$.

Proof. To verify (1) we first use $[F F, 3.8]$ to infer that $f_{*}(T \cap V)=C \psi$, where $\psi$ is an integer valued function vanishing outside $W$. Recalling the last statement in $[\mathrm{FF}, 2.3]$ we then prove that $\psi$ is constant on $W$, by showing that

$$
\text { spt } \partial f_{\#}(T \cap V) \subset R^{n}-W .
$$

If $\phi \in E^{n-1}\left(R^{n}\right)$ and $\operatorname{spt} \phi \subset W$, then the sets

$$
V \cap f^{-1}(\operatorname{spt} \phi) \text { and }(\operatorname{spt} T)-V
$$

are compact and disjoint, hence there exists $u \in E^{0}(X)$ such that

$$
\begin{aligned}
u(x) & =1 \text { whenever } x \in V \cap f^{-1}(\operatorname{spt} \phi), \\
u(x) & =0 \text { whenever } x \in(\operatorname{spt} T)-V, \\
d u(x) & =0 \text { whenever } x \in(\operatorname{spt} T) \cap f^{-1}(\operatorname{spt} \phi) ;
\end{aligned}
$$

inasmuch as $f^{-1}(\operatorname{spt} \phi) \subset f^{-1}(W) \subset X-\operatorname{spt}(\partial T)$, it follows that

$$
\begin{aligned}
f_{*}(T \cap V)[d \phi] & =T\left[V \cap d f^{*}(\phi)\right]=T\left[u \wedge d f^{*}(\phi)\right] \\
& =T\left[-d u \wedge f^{*}(\phi)\right]=0 .
\end{aligned}
$$


Next we observe that (2) is obvious, and implies (3). To obtain (4) we compute

where $K=\Gamma(W) \cap\left\{V: V \subset V^{\prime}\right\}$.

$$
\begin{aligned}
\Delta\left(V^{\prime}\right) \cdot W & =f_{*}\left(T \cap V^{\prime}\right) \cap W=f_{*}\left[T \cap V^{\prime} \cap f^{-1}(W)\right] \\
& =f_{*}[\bigcup K]=\sum_{V \in K} \Delta(V) \cdot W,
\end{aligned}
$$

To prove (5) suppose $x \in \operatorname{spt} T-\operatorname{spt} \partial T$, choose sets $W_{1} \supset W_{2} \supset W_{3} \supset \cdots$ in $G$ whose closures have the intersection $\{f(x)\}$, and choose $V_{j} \in \Gamma\left(W_{j}\right)$ so that $x \in V_{j}$. The closures of the sets $V_{j}$ are continua whose intersection is a continuum contained in the finite set $f^{-1}\{f(x)\}$, hence equals $\{x\}$.

To obtain (6) we choose $W^{\prime} \in G$ and $V^{\prime} \in \Gamma\left(W^{\prime}\right)$ so that $V^{\prime} \cap f^{-1}\{f(x)\}=\{x\}$. For any $V^{\prime \prime} \in H$ such that $x \in V^{\prime \prime} \subset V^{\prime}$, say $V^{\prime \prime} \in \Gamma\left(W^{\prime \prime}\right)$, we let $W=W^{\prime} \cap W^{\prime \prime}$, note that $V^{\prime \prime}$ belongs also to $\Gamma(W)$, and infer from (4) and (2) that $\Delta\left(V^{\prime}\right)=\Delta\left(V^{\prime \prime}\right)$.

To prove (7) we choose $F$ according to [FF, 8.16], let

$$
B=(\bigcup F) \cap[\operatorname{spt} T-\operatorname{spt} \partial T] \cap f^{-1}(E),
$$

and infer with the help of the classical area formula $[F 1,5.9]$ that

$$
\begin{aligned}
M\left[T \wedge f^{*}\left(\omega_{n} \cap E\right)\right] & \\
& =\int_{f-1(E)}\left|f^{*}\left(\omega_{n}\right)[\vec{T}(x)]\right| \Theta^{n}(\|T\|, x) d H^{n} x \\
& \leqq \mu \int_{B}\left|f^{*}\left(\omega_{n}\right)[\vec{T}(x)]\right| d H^{n} x \\
& =\mu \int_{R^{n}} N(f, B, y) d L_{n} y \leqq \mu \nu L_{n}(E) .
\end{aligned}
$$

Finally, to verify (8) and (9), we fix $y \in R^{n}-f(\operatorname{spt} \partial T)$ and $\varepsilon>0$, and abbreviate (spt $T) \cap f^{-1}\{y\}=A$. Then $y$ has a neighborhood $W^{\prime} \in G$ such that the sets $V_{x}^{\prime} \in \Gamma\left(W^{\prime}\right)$ corresponding to $x \in A$, for which $x \in V_{x}^{\prime}$, satisfy the conditions

$$
\operatorname{diam}\left(V_{x}^{\prime}\right)<\varepsilon \text { and } \Delta\left(V_{x}^{\prime}\right)=\Delta(x) .
$$

We note that the set

$$
Z=W^{\prime} \cap\left\{z: f^{-1}\{z\} \subset \bigcup_{x \in A} V_{x}^{\prime}\right\}
$$

is a neighborhood of $y$ in $R^{n}$.

Taking any $\psi \in E^{0}(X)$ with $F(\psi) \leqq 1$, we define the function $\gamma$ on $\bigcup\left\{V_{x}^{\prime}: x \in A\right\}$ by setting

$$
\gamma(v)=\psi(x) \text { whenever } v \in V_{x}^{\prime},
$$

whence $|\gamma(v)-\psi(v)| \leqq \varepsilon$.

If $W \subset Z$ and $W \in G$, then (7) yields 
Moreover

$$
\left|\left\langle T, f, \omega_{n} \cap W\right\rangle(\psi-\gamma)\right| \leqq \varepsilon \mu \nu L_{n}(W)
$$

$$
\begin{aligned}
& \left\langle T, f, \omega_{n} \cap W\right\rangle(\gamma)=T\left[f^{\#}\left(\vec{\omega}_{n}\right) \wedge \gamma \cap f^{-1}(W)\right] \\
& =\sum_{x \in A} \psi(x) T\left[f^{*}\left(\omega_{n}\right) \cap V_{x}^{\prime} \cap f^{-1}(W)\right] \\
& =\sum_{x \in A} \psi(x) f_{\#}\left(T \cap V_{x}^{\prime}\right)\left[\omega_{n} \cap W\right] \\
& =\sum_{x \in A} \psi(x) \Delta(x) L_{n}(W)
\end{aligned}
$$

and we obtain the inequality

$$
\left|\left\langle T, f, \omega_{n} \cap W\right\rangle(\psi)-L_{n}(W) \sum_{x \in A} \Delta(x) \psi(x)\right| \leqq \varepsilon \mu \nu L_{n}(W)
$$

We can immediately generalize this inequality, replacing $W \in G$ by an arbitrary Borel subset of $Z$; then (8) and (9) become obvious.

4. Complex varieties as minimal currents. Following a simple proof of Wirtinger's inequality it is shown that, in a Kähler manifold, integral currents with almost everywhere complex tangent spaces are minimal currents. Then (locally) integral currents are associated with complex algebraic varieties, through integration over the nonsingular part. It is proved that the plane sections of a variety, which are defined algebrogeometrically but which also correspond to slices in the sense of $\S 3$, vary continuously as currents. This fact is used in the final theorem, concerning the tangent cones of complex algebraic varieties.

Basic information on Kähler manifolds and algebraic varieties may be found in [WK] and [SM].

4.1 TheORem. Suppose $V$ is a complex vectorspace with a positive definite Hermitian form

$$
H=S+i A,
$$

where $S$ and $A$ are symmetric and alternating real bilinear functions, respectively. Consider $V$ as a real vectorspace with the inner product $S$, and with the alternating forms

$$
B_{r}=A^{r} / r !
$$

of degree $2 r$, where $A^{r}$ is the r-fold exterior product of $A$.

If $w_{1}, \cdots, w_{2 r}$ are mutually orthogonal unit vectors in $V$, then

$$
B_{r}\left(w_{1}, \cdots, w_{2 r}\right) \leqq 1 .
$$

Moreover, equality holds if and only if the real vectorspace spanned by $w_{1}, \cdots, w_{2 r}$ is in fact a complex vector subspace of $V$, and its natural orientation is $w_{1}$ $w_{1} \wedge \cdots \wedge w_{2 r}$. 
Proof. First consider the case $r=1$. If $u$ and $v$ are orthogonal unit vectors in $V$, then

$$
H(u, v)=i A(u, v),
$$

hence Schwarz's inequality yields $A(u, v) \leqq 1$, with equality if and only if $H(u, v)=i$, which is equivalent to $v=i u$.

Proceeding to the general case, suppose $W$ is any real $2 r$ dimensional vector subspace of $V$, and $\Phi$ is the restriction of $A$ to $W$. Then $W$ has an orthonormal base $e_{1}, \cdots, e_{2 r}$ with the dual base $\phi_{1}, \cdots, \phi_{2 r}$ such that

$$
\Phi=\sum_{j=1}^{r} \lambda_{j}\left(\phi_{2 j-1} \wedge \phi_{2 j}\right)
$$

where $\lambda_{j}=A\left(e_{2 j-1}, e_{2 j}\right)$. It follows that

$$
\begin{gathered}
\Phi^{r}=r ! \bigwedge_{j=1}^{r} \lambda_{j}\left(\phi_{2 j-1} \wedge \phi_{2 j}\right), \\
B_{r}\left(e_{1}, \cdots, e_{2 r}\right)=\prod_{j=1}^{r} \lambda_{j} .
\end{gathered}
$$

Applying the special case first considered to each pair $\left(e_{2 j-1}, e_{2 j}\right)$ we find that

$$
\prod_{j=1}^{r} \lambda_{j} \leqq 1
$$

and that equality holds if and only if $\lambda_{j}= \pm 1$ for each $j$, with minus occurring an even number of times; moreover each minus can be made a plus by interchanging $e_{2 j-1}$ and $e_{2 j}$.

4.2 THEOREM. If $X$ is a Kähler manifold with the fundamental form $\Omega \in E^{2}(X)$ and if $T \in I_{2 r}(X)$, then:

(1) $T\left(\Omega^{r} / r\right.$ !) $M(T)$. Moreover, equality holds if and only if for $\|T\|$ almost all $x$ in $X$ the oriented $2 r$ dimensional real vectorspace of $\vec{T}(x)$ is a naturally oriented complex subspace of the tangentspace of $X$ at $x$.

(2) In case $T\left(\Omega^{r} / r\right.$ !) $=M(T)$, then

$$
M(T) \leqq M(T+\partial U) \text { whenever } U \in I_{2 r+1}(X) .
$$

(3) In case $T\left(\Omega^{r} / r !\right)=M(T)$ and $\Omega^{r} / r !=d \Psi$ for some $\Psi \in E^{2 r-1}(X)$, then

$$
M(T) \leqq M(Z) \text { whenever } Z \in I_{2 r}(X) \text { and } \partial Z=\partial T \text {. }
$$

Proof. Since

$$
M(T)-T\left(\Omega^{r} / r !\right)=\int_{X}\left(1-\frac{\Omega^{r}}{r !}[\vec{T}(x)]\right) d\|T\| x,
$$

we obtain (1) by applying Theorem 4.1 to each tangentspace of $X$.

To verify (2), note that 


$$
\begin{aligned}
M(T+\partial U) & \geqq(T+\partial U)\left(\Omega^{r} / r !\right) \\
& =M(T)+U\left(d \Omega^{r} / r !\right)=M(T)
\end{aligned}
$$

because $d \Omega=0$.

Finally, (3) holds because

$$
\begin{aligned}
M(Z)-M(T) & \geqq Z\left(\Omega^{r} / r !\right)-T\left(\Omega^{r} / r !\right) \\
& =(\partial Z)(\Psi)-(\partial T)(\Psi)=0 .
\end{aligned}
$$

4.3 Remark. Clearly (3) implies that $T$ is a current of least mass [FF, 9.1]; it is applicable in case $X=C^{n}$. Moreover (2) implies that $T$ is a minimal current, because all cycles with small supports are boundaries; it is applicable in case $X=P^{n}(C)$.

4.4 LoCAlly NORMAL CURRENTS. Following [DR1] we let $D^{k}(X)$ be the topological vectorspace of all real valued $k$ forms of class $\infty$ on $X$ with compact support, and $\boldsymbol{D}_{\boldsymbol{k}}(X)$ the dual space of all real valued $k$ dimensional currents, whose support need not be compact.

Assuming $T \in D_{k}(X)$, we call $T$ locally normal if and only if for each $x \in X$ there exists a normal current $S \in E_{k}(X)$ such that $x \notin \operatorname{spt}(T-S)$. It is obvious that, on each compact subset of $X$, locally normal currents behave just like normal currents. For instance, while $M(T)$ may be infinite, the measure $\|T\|$ remains finite on compact sets. If $f: X \rightarrow Y$ is locally Lipschitzian, then locally normal currents $\langle T, f, \phi\rangle$ and $\langle T, f, y\rangle$ may be defined essentially the same way as when $T$ is normal; moreover $f_{*}(T)$ may be defined in case the restriction of $f$ to spt $T$ is proper.

Similarly we define locally rectifiable and locally integral currents.

4.5 Complex Algebraic VARIETIES AS CURRENTS( $\left.{ }^{4}\right)$. Suppose $V$ is a complex $r$ dimensional irreducible algebraic variety in complex $n$ space $C^{n}$. We shall verify first, by induction with respect to $r$, that

$$
H^{2 r}(V \cap B)<\infty \text { for every bounded Borel set } B \subset C^{n} .
$$

Since the singular subset $S$ of $V$ is a complex algebraic set of dimension $\leqq r-1$, we may assume that $H^{2 r-2}(S \cap B)<\infty$ for every bounded Borel set $B \subset C^{n}$, whence

$$
H^{2 r-1}(S)=0 \text {. }
$$

We recall that $V-S$ is a connected complex $r$ dimensional analytic submanifold of $C^{n}$, and that the Kähler form of $C^{n}$ is given by the formula

$$
\Omega=(2 i)^{-1} \sum_{j=1}^{n} d Z_{j} \wedge d Z_{j}=\sum_{j=1}^{n} Z_{j}^{*}\left(\omega_{2}\right)
$$

(4) Complex varieties have been previously studied as currents in [ST], [L], [DR 2]. The irrelevance of the singular subset is proved here by a new method. 
where $Z_{j}: C^{n} \rightarrow C \simeq R^{2}$ is the $j$ th complex coordinate function. Consequently

$$
\Omega^{r} / r !=\sum_{\lambda \in \Lambda(k, n)} q^{\lambda *}\left(\omega_{2 r}\right)
$$

where $q^{\lambda}: C^{n} \rightarrow C^{r} \simeq R^{2 r}, q^{\lambda}(z)=\left(z_{\lambda(1)}, \cdots, z_{\lambda(r)}\right)$ for $z \in C^{n}$, and we use 4.1 and the classical area formula to compute

$$
\begin{aligned}
H^{2 r}(V \cap B) & =\int_{V \cap B-S} \Omega^{r} / r ! \\
& =\sum_{\lambda \in \Lambda(k, n)} \int N\left(q^{\lambda}, V \cap B-S, y\right) d L_{2 r} y \\
& \leqq \sum_{\lambda \in \Lambda(k, n)} \operatorname{degree}(V) \cdot L_{2 r}\left[q^{\lambda}(B)\right]<\infty .
\end{aligned}
$$

We may now define a locally rectifiable current $T \in D_{2 r}\left(C^{n}\right)$ by the formula

$$
T(\phi)=\int_{V-s} \phi \quad \text { for } \phi \in D^{2 r}\left(C^{n}\right) .
$$

Clearly spt $T \subset V$ and spt $\partial T \subset S$, and we see from 2.2 (localized, and with $U=0$ ) that

$$
\partial T=0 .
$$

Hereafter we shall not distinguish notationally between the affine variety $V$ and the corresponding locally integral current $T$. Similarly we may regard a complex $r$ dimensional algebraic variety in the projective space $P^{n}(C)$ as a member of $I_{2 r}\left[P^{n}(C)\right]$. Of course the usual nonhomogeneous coordinate systems do not map the Kähler form of $C^{n}$ onto the Kähler form of $P^{n}(C)$, but they do map locally integral currents onto locally integral currents, and preserve all local convergence properties of currents.

Whenever the intersection $V \bullet W$ of two algebraic varieties $V$ and $W$, with complex dimensions $r$ and $s$, exists in the sense of algebraic geometry, then $V \bullet W$ is a linear combination of complex $r+s-n$ dimensional varieties with positive integral coefficients, hence will be regarded as a (locally) integral current of dimension $2(r+s-n)$.

4.6 Remark. Suppose $V$ is a complex $r$ dimensional irreducible algebraic variety in $P^{n}(C)$ and $W$ is a complex $s$ dimensional projective subspace of $P^{n}(C)$ such that $V \bullet W$ exists. Letting

$$
\begin{aligned}
& \rho: C^{n} \rightarrow P^{n}(C), \quad \rho(z)=\left(z_{1}: z_{2}: \cdots: z_{n}: 1\right), \\
& f: C^{n} \rightarrow C^{n-s}, \quad f(z)=\left(z_{1}, z_{2}, \cdots, z_{n-s}\right),
\end{aligned}
$$

we assume that $\rho^{-1}(W)=f^{-1}\{0\}$ and that $V \cap W-\rho\left(C^{n}\right)$ has dimension less than $r+s-n$. We shall eventually prove the formula 


$$
V \bullet W=\rho_{\#}\left(\left\langle f, \rho^{-1}(V), 0\right\rangle\right) .
$$

In the special case $s=n-r$, this formula becomes obvious when one combines 3.17 with the classical definition of algebraic intersection multiplicity. In fact one may assume (after replacing $Z_{r+1}$ by $Z_{r+1}+\sum_{j=r+2}^{n} \quad c_{j} Z_{j}$ for suitable $c_{j} \in C$ ) that $Z_{r+1}$ is one-to-one on $\rho^{-1}(V \cap W)$, and that the function field of $\rho^{-1}(V)$ is generated by the images $\zeta_{1}, \cdots, \zeta_{r+1}$ of $Z_{1}, \cdots, Z_{r+1}$. If $F$ is an irreducible polynomial such that $F\left(\zeta_{1}, \cdots, \zeta_{r+1}\right)=0$, and if the point $\xi$ occurs with multiplicity $m(\xi)$ in $V \bullet W$, then $m(\xi)$ equals the multiplicity of $\xi_{r+1}$ as a root of $F\left(0, \cdots, 0, Z_{r+1}\right)$, and $L_{2 r}$ almost all points of $C^{r}$ near 0 have $m(\xi)$ counterimages near $\xi$ on $\rho^{-1}(V)$, with respect to $f$, hence $\Delta(\xi)=m(\xi)$.

This special case will be applied in the proof of Theorem 4.7, which will in turn be used for obtaining the general formula in 4.8 .

4.7 THEOREM. Suppose $r+s \geqq n, V$ is a complex $r$ dimensional algebraic variety in the $n$ dimensional projective space $P^{n}(C)$, and $G$ is the set of those complex $s$ dimensional projective subspaces $W$ of $P^{n}(C)$ for which the intersection $V \bullet W$ exists. Then the function mapping $W \in G$ onto $V \bullet W \in I_{2(r+s-n)}\left[P^{n}(C)\right]$ is continuous with respect to the $\boldsymbol{F}$ topology.

Proof. In the special case when $r+s=n$, this theorem is essentially well known; it is merely a reformulation of the classical principle that the roots of a system of algebraic equations depend continuously on the coefficients.

Proceeding to the case when $r+s>n$, suppose $W_{1}, W_{2}, W_{3}, \cdots \in G$ converge to $W \in G$, and let

$$
V \bullet W=\sum_{k=1}^{m} \sigma_{k} Q_{k}
$$

where $\sigma_{1}, \cdots, \sigma_{m}$ are positive integers and $Q_{1}, \cdots, Q_{m}$ are complex $r+s-n$ dimensional irreducible varieties, the algebraic components of $V \cap W$. Also let $S$ be the subset of $V \cap W$ consisting of all those points which either belong to more than one $Q_{k}$, or are singular points of some $Q_{k}$. Note that each $Q_{k}-S$ is a connected complex variety.

Since degree $\left(V \bullet W_{j}\right)=\operatorname{degree}(V)$ for all $j$, the sequence of integral cycles $V \bullet W_{j}$ is $M$ bounded. Therefore, according to [FF 8.13, 7.3], this sequence has subsequences which are $\boldsymbol{F}$ convergent to integral cycles. It will be sufficient to show that the limit of each such convergent subsequence must be $V \bullet W$.

Replacing the given sequence by a subsequence, we henceforth assume the existence of a current $T \in I_{2(r+s-n)}\left[P^{n}(C)\right]$ for which

$$
\lim _{j \rightarrow \infty} F\left(V \bullet W_{j}-T\right)=0 .
$$

Since spt $T \subset V \cap W, \partial T=0$ and $H^{2(r+s-n)}(S)=0$, there exist integers $\tau_{1}, \cdots, \tau_{m}$ such that 


$$
T=\sum_{k=1}^{m} \tau_{k} Q_{k} .
$$

In view of 3.8 and 4.6 we may choose a complex

$$
n-(r+s-n)=2 n-r-s
$$

dimensional projective subspace $E$ of $P^{n}(C)-S$ such that

$$
\liminf _{j \rightarrow \infty} F\left[\left(V \bullet W_{j}\right) \bullet E-T \bullet E\right]=0
$$

and $W_{j} \cap E, W \cap E$ are complex projective spaces of dimension $n-s$. Using the associative law, and the special case of the theorem mentioned initially, we find that also

$$
\lim _{j \rightarrow \infty} F\left[V \bullet\left(W_{j} \bullet E\right)-V \bullet(W \bullet E)\right]=0,
$$

hence $T \bullet E=(V \bullet W) \bullet E$.

Finally we choose $\psi_{k} \in E^{0}\left[P^{n}(C)\right]$ so that

$$
\begin{aligned}
& \psi_{k}(x)=1 \text { whenever } x \in E \cap Q_{k}, \\
& \psi_{k}(x)=0 \text { whenever } x \in Q_{t} \text { with } t \neq k,
\end{aligned}
$$

and conclude that

$$
\begin{aligned}
\tau_{k} \text { degree }\left(Q_{k} \bullet E\right) & =(T \bullet E)\left(\psi_{k}\right) \\
& =[(V \bullet W) \bullet E]\left(\psi_{k}\right)=\sigma_{k} \operatorname{degree}\left(Q_{k} \bullet E\right),
\end{aligned}
$$

hence $\tau_{k}=\sigma_{k}$.

4.8 REMARK. We shall now see why the formula stated in 4.5 holds also in case $s>n-r$. Letting

$$
\begin{array}{ll}
g: C^{n} \rightarrow C^{r+s-n}, & g(z)=\left(z_{n-s+1}, \cdots, z_{r}\right), \\
h: C^{n} \rightarrow C^{r}, & h(z)=\left(z_{1}, \cdots, z_{r}\right),
\end{array}
$$

we infer from 4.6, 3.12 (3) and the associative law that, for $L_{2 r}$ almost all $(u, v)$ in $C^{n-s} \times C^{r+s-n}$,

$$
\begin{aligned}
\left\langle\rho^{-1}(V), f, u\right\rangle & g^{-1}\{v\} \\
= & \left\langle\left\langle\rho^{-1}(V), f, u\right\rangle, g, v\right\rangle \\
& =\left\langle\rho^{-1}(V), h,(u, v)\right\rangle=\rho^{-1}(V) \bullet h^{-1}\{(u, v)\} \\
& =\rho^{-1}(V) \bullet\left[f^{-1}\{u\} \bullet g^{-1}\{v\}\right]=\left[\sigma^{-1}(V) \bullet f^{-1}\{u\}\right] \bullet g^{-1}\{v\} .
\end{aligned}
$$

It follows that the formula

$$
\left\langle\sigma^{-1}(V), f, u\right\rangle=\rho^{-1}(V) \bullet f^{-1}\{u\}
$$


holds for $L_{2 n-2 s}$ almost all $u$ in $C^{n-s}$. Moreover the preceding theorem implies that the right member is continuous with respect to $u$, wherever it exists. Then 3.5 and 3.6(3) show that the formula holds whenever $\rho^{-1}(V) \bullet f^{-1}\{u\}$ exists.

4.9 TANGent CONES. By a $k$ dimensional cone in $R^{m}$, with vertex at the origin 0 , we mean here a current $0 S$ (see $[F F, 9.2]$ ) where $S \in I_{k-1}\left(R^{m} \cap\{x:|x|=1\}\right)$. Such a cone is called the measuretheoretic tangent cone of a $k$ dimensional locally integral current $T$ at 0 if and only if it equals the $F$ limit, as $\varepsilon \rightarrow 0+$, of the currents

$$
T=\mu_{1 / \ell *}(T) \cap\{x:|x|<1\}
$$

where $\mu_{1 / \varepsilon}(x)=x / \varepsilon$ for $x \in R^{m}$. The problem discussed in [FF, 9.2], whether the measuretheoretic tangent cone always exists in case $T$ is a minimal current, is still unsolved.

Now suppose $T$ is a complex $r$ dimensional algebraic variety in $C^{n}$, with $0 \in T$. Choosing a generic point $\zeta=\left(\zeta_{1}, \cdots, \zeta_{n}\right)$ of $T$ over $C$, and a quantity $t$ transcendental over the field $C(\zeta)$, one considers the complex $r+1$ dimensional variety $V$ in $C^{n+1}$ with the generic point $\left(\zeta_{1} / t, \cdots, \zeta_{n} / t, t\right)$, and the complex $n$ dimensional plane

$$
W=C^{n+1} \cap\left\{w: w_{n+1}=0\right\} \text {. }
$$

The intersection $V \bullet W$ exists. Using the projection $p: C^{n+1} \rightarrow C^{n}, p(w)=\left(w_{1}, \cdots, w_{n}\right)$, one may define the algebrogeometric tangent cone of $T$ at 0 as the current

$$
p_{*}(V \bullet W) \cap\{z:|z|<1\} \text {. }
$$

In all these definitions, the restriction to the unit ball is only a device yielding currents of finite mass, and is not essential.

4.10 THEOREM. The algebrogeometric and measuretheoretic tangent cones of a complex algebraic affine variety coincide at every point.

Proof. We readopt the notation of 4.9 , let

$$
q: C^{n+1} \rightarrow C, \quad q(w)=w_{n+1},
$$

and choose $\psi \in D_{0}(C)$ so that $\psi(\varepsilon)=1$ for all $\varepsilon$ near 0 . Note that, if $\varepsilon \neq 0$, then

$$
\begin{array}{r}
V \bullet q^{-1}\{\varepsilon\}=\mu_{1 / \varepsilon *}(T) \times\{\varepsilon\}, \\
p_{*}\left(V \bullet q^{-1}\{\varepsilon\}\right)=\mu_{1 / \ell *}(T) .
\end{array}
$$

Since the planes $q^{-1}\{\varepsilon\}$ converge to $q^{-1}\{0\}=W$ as $\varepsilon \rightarrow 0$, it follows from 4.7 that, for each $\phi \in D^{2 r}\left(C^{n}\right)$,

$$
\begin{aligned}
p_{*}(V \bullet W)(\phi) & =(V \bullet W)\left[p^{*}(\phi) \wedge q^{*}(\psi)\right] \\
& =\lim _{\varepsilon \rightarrow 0}\left(V \bullet q^{-1}\{\varepsilon\}\right)\left[p^{*}(\phi) \wedge q^{*}(\psi)\right] \\
& =\lim _{\varepsilon \rightarrow 0} \mu_{1 / \varepsilon *}(T)(\phi) .
\end{aligned}
$$




\section{BIBLIOGRAPHY}

F. J. Almgren, JR.

[A] The homotopy groups of the integral cycle groups, Topology 1 (1962), 257-299.

E. DE GIORGI

[DG] Frontiere orientate di misura minima, Seminaria di Matematica Scuola Norm. Sup. Pisa, 1960-61.

G. De RhaM

[DR. 1] Variétés différentiables, Actualités Sci. Ind. No. 1222, Hermann, Paris, 1955.

[DR. 2] On the area of complex manifolds, Seminar on several complex variables, Institute for Advanced Study, Princeton, N.J., 1957.

H. FEDERER and W. H. FLEMING

[FF] Normal and integral currents, Ann. of Math. (2) 72 (1960), 458-520.

H. FEDERER

[F1] The $(\varphi, k)$ rectifiable subsets of $n$ space, Trans. Amer. Math. Soc. 62 (1947), 114-192.

[F2] Some integralgeometric theorems, Trans. Amer. Math. Soc. 77 (1954), 238-261.

[F3] Curvature measures, Trans. Amer.Math. Soc. 93 (1959), 418-491.

[F4] Currents and area, Trans. Amer. Math. Soc. 98 (1961), 204-233.

[F5] Approximation of integral currents by cycles, Proc. Amer. Math. Soc. 12 (1961), 882-884.

W. H. FLEMING

[FL] On the oriented Plateau problem, Rend. Circ. Mat. Palermo (2) 11 (1962), 69-90.

P. LELONG

[L] Intégration sur un ensemble analytique complexe, Bull. Soc. Math. France 85(1957), 239-262.

J. H. MichaEL

[M] The convergence of measures on parametric surfaces, Trans. Amer. Math. Soc. 107 (1963), 140-152.

E. R. REIFENBERG

[R] An epiperimetric inequality related to the analyticity of minimal surfaces, Ann. of Math. (to appear).

S. SAKS

[S] Theory of the integral, Warsaw, 1937.

P. SAMUEL

[SM] Méthodes d'algèbre abstraite en géométric algébrique, Vol. 4, Ergebnisse der Mathematik und ihrer Grenzgebiete, Springer, Berlin, 1955.

W. STOLL

[ST] Mehrfache Integrale auf komplexen Mannigfaltigkeiten, Math. Z. 57 (1952), 116-154.

A. WeIL

[WK] Introduction à l'étude des variétés Kähleriennes, Actualités Sci. Ind. No.1267, Hermann, Paris, 1955.

W. WIRTINGER

[W] Eine Determinantenidentität und ihre Anwendung auf analytische Gebilde in Euclidischer und Hermitescher Massbestimmung, Monatsh. Math. Phys. 44 (1936), 343-365.

W. P. ZIEMER

[Z] Integral currents mod 2, Trans. Amer. Math. Soc. 105 (1962), 496-524.

BROWN UNIVERSITY,

Providence, Rhode IsLand 